\title{
O Ensino sobre Deficiência a Estudantes de Medicina: o que Existe no Mundo?
}

\author{
Teaching Medical Students about \\ Disability: What Is There in the World?
}

\section{PALAVRAS-CHAVE \\ - Educação Médica; \\ - Pessoas com Deficiências; \\ - Atenção à Saúde.}




\section{INTRODUÇÃO}

A formação de profissionais de saúde, especialmente de médicos, foco do estudo aqui relatado, deveria ser uma preocupação de todas as escolas de Medicina no que diz respeito a: promoção do conhecimento sobre as necessidades em saúde das Pessoas com Deficiência (PCD) e como atendê-las, o estímulo a atitudes positivas em relação às pessoas desse grupo $\mathrm{e}$ o desenvolvimento das habilidades necessárias à prestação do cuidado em saúde às $\mathrm{PCD}$, nas diversas disciplinas, teóricas e práticas, assim como nos programas de internato e residência, segundo os princípios dos direitos humanos ${ }^{1}$.

Na década de 1970, a Organização Mundial da Saúde estimou em $10 \%$ o percentual de pessoas com deficiência no mundo, fora de períodos de guerra. Hoje, essa estimativa passou a ser de $15 \%{ }^{1}$, principalmente por conta do envelhecimento da população mundial e da crescente prevalência de doenças crônicas. Também contribuem para essa cifra os acidentes de trânsito, doenças ocupacionais e crises humanitárias. As pessoas com deficiência apresentam necessidades de cuidados gerais (imunização, exames preventivos, tratamento de doenças crônicas e agudas) e de cuidados específicos, em fisioterapia, fonoaudiologia, psicologia, terapia ocupacional.

As PCD apresentam tabagismo, sobrepeso, consumo de álcool e outras drogas, depressão e inatividade física numa frequência superior à de pessoas sem deficiência ${ }^{1}$. Publicações recentes, entretanto, apontam falhas na atenção às necessidades de saúde das PCD em praticamente todo o mundo, denunciando uma atenção ainda distante das dimensões do cuidado de saúde de qualidade: medicina centrada no paciente; segurança; eficácia; que não haja longas esperas para a consulta; eficiência e equidade ${ }^{2}$.

Em 2002, a Política Nacional de Saúde da Pessoa com Deficiência $^{3}$ sinalizava que seriam desenvolvidas ações conjuntas com o Ministério da Educação e com as Instituições de Ensino Superior, considerando a necessidade de incorporar disciplinas e conteúdos de reabilitação e atenção à saúde das PCD nos currículos de graduação na área da saúde, sendo que até agora não houve nenhum movimento nesse sentido. As Diretrizes Curriculares Nacionais para o Ensino de Graduação em Medicina, instituídas pela Câmara de Educação Superior do Conselho Nacional de Educação ${ }^{4}$, não especificam atenção às pessoas com deficiência, embora as competências e habilidades que se esperam que os estudantes adquiram ao longo do curso devam ser estendidas a este grupo. Basta focar a atenção nos itens do Artigo $5^{\circ}$ para perceber a enorme lacuna da formação médica no Brasil para atender às competências e habilidades específicas voltadas à atenção à saúde das PCD.
No curso médico da Universidade Federal Fluminense (UFF), o processo de inclusão de conteúdos em atenção à saúde das pessoas com deficiência teve início em 2004, nas áreas de ensino, pesquisa e extensão ${ }^{5-12}$. O ensino é pautado pelos referenciais: modelo biopsicossocial de perceber a deficiência; inclusão de pessoas com deficiência como educadores e no planejamento de atividades; integralidade na atenção à saúde; saúde como um direito; e pessoas com deficiência fazendo parte da diversidade humana.

De modo a avaliar e ampliar nossa prática docente no ensino de tais temas, buscamos conhecer outros exemplos de inclusão do tema Deficiência no curso médico que possam servir de inspiração para o trabalho desenvolvido na UFF e ponto de partida para novos projetos de inclusão ou ampliação de projetos já em andamento em outras universidades.

\section{MATERIAL E MÉTODOS}

\section{Material}

Artigos relacionados à inclusão do tema deficiência nos currículos de Medicina publicados entre 2000 e 2011.

\section{Método}

A pesquisa se iniciou nos primeiros meses de 2012. Assim, foram levantados artigos publicados de 2000 até 2011, através do Google Scholar e Pubmed. Nesse período, esperava-se abranger uma boa diversidade de experiências e um número razoável de artigos para analisar. O sumário de todos os números da Revista da Associação Brasileira de Educação Médica desse período foi pesquisado. Foram selecionados apenas os periódicos científicos, e todos os artigos citados foram lidos na íntegra.

Com o objetivo de identificar experiências de ensino relativas à saúde das pessoas com deficiência, foram escolhidos os seguintes descritores: People with Disability; Medical Education; Teaching; Disability; e Medical Students.

Foram descartados os artigos que se referiam, por exemplo, ao ensino de reabilitação e os que apresentavam experiências de ensino na pós-graduação. Embora para a análise tenham sido incluídos apenas aqueles que descreviam as atividades desenvolvidas junto aos estudantes, foram lidos também aqueles que traziam informações gerais quanto ao ensino sobre deficiência em todo o país de referência, e outros que sugeriam conteúdos a serem incluídos. Outros artigos foram selecionados com base nas referências bibliográficas dos artigos lidos, mesmo que anteriores a 2000. Ao todo, dos 82 artigos selecionados, foram utilizados 50 (61\%).

Após leitura flutuante de todos os artigos pelas duas autoras, cada uma delas realizou um fichamento, separadamente. A 
busca cessou quando houve a saturação das contribuições, consensual. O conteúdo dos artigos foi organizado em cinco categorias: (1) período do curso em que ocorreu a inserção; (2) espaço do curso onde a(s) atividade(s) foram realizadas; (3) estratégias educacionais; (4) objetivo da atividade; (5) conteúdo; (6) participação de pessoas com deficiência, seus familiares e cuidadores.

\section{RESULTADOS}

O período de inserção da atividade no currículo variou bastante: no primeiro ano ${ }^{13}$; primeiro e segundo ${ }^{14}$; segundo ano ${ }^{15}$; terceiro $^{16,17}$; e no quarto ${ }^{18}$. Na Universidade de Buffalo, a implementação foi planejada para todos os quatro anos do currículo $^{19}$. Nos primeiros anos do curso, as atividades se voltam à promoção de atitudes positivas dos estudantes de Medicina em relação às pessoas com deficiência; à aquisição de conhecimento acerca dos direitos desse grupo; ao modelo social de perceber a deficiência; à dissociação entre deficiência e doença; ao desenvolvimento de habilidades de comunicação com pessoas com deficiência, e a outros. No curso de Desenvolvimento Pessoal e Profissional da Universidade de Leeds, no Reino Unido, foi incluída a conscientização sobre a surdez e o modelo social de perceber a deficiência ${ }^{20}$.

Já nos últimos anos, a ênfase recai sobre o desenvolvimento de habilidades no atendimento a pacientes com deficiência no nível de atenção primária, em atividades tanto de ensino quanto de avaliação dos estudantes, incluindo anamnese e exame físico. Nos três primeiros anos do curso médico da Universidade de Newcastle, na Austrália, são apresentados casos clínicos que trazem aspectos da deficiência física entre os problemas clínicos apresentados ${ }^{21}$.

De modo geral, as atividades nos últimos anos do curso fazem parte de módulos das áreas de Medicina da Família, Pediatria e Psiquiatria ${ }^{17,22-26}$. Assim, o objetivo da atividade pode ser a promoção de atitudes positivas dos estudantes em relação às pessoas com deficiência, a construção de conhecimentos ou o desenvolvimento de habilidades. Alguns exemplos se dirigiram a apenas um desses objetivos; outros, a dois; com menos frequência, aos três.

Para seleção do conteúdo, algumas escolas organizaram painéis para identificar habilidades, conhecimentos e atitudes que seriam essenciais e os desejáveis no currículo médico, como na Universidade de Bristol, no Reino Unido ${ }^{27}$. Foram apontadas como essenciais: explorar as próprias atitudes e sentimentos; conhecer o lugar das pessoas com deficiência na sociedade (atitudes); comunicação com pessoas com deficiência auditiva, visual, da fala e cognitiva (habilidades). No curso médico de Wisconsin (Milwaukee), estudantes receberam treinamento em etiqueta a ser seguida nos encontros com pa- cientes com deficiência ${ }^{28}$, e para apresentarem habilidade em posicionar e movimentar pessoas que apresentam hemiplegia, no leito ou em cadeira de rodas ${ }^{16}$. Embora essa habilidade seja promovida em cursos de fisioterapia e enfermagem, isso não acontece no curso médico, apesar de sua importância para o exame do paciente nessa situação e de fácil ensino.

As estratégias educacionais variaram entre as seguintes: aulas quando o objetivo era promover o conhecimento dos estudantes $^{29,23}$; seminários ${ }^{20}$; painéis ${ }^{17}$; oficinas ${ }^{24,27}$; anamneses $^{25,30,31,32}$; visitas a serviços que atendem pessoas com deficiência ${ }^{22,33}$; e vivências onde os estudantes eram desafiados a realizar atividades usando cadeiras de rodas, muletas, vendas nos olhos ${ }^{14,34}$. French ${ }^{35}$ critica o uso isolado de vivências por não haver evidência de que tenham efeito positivo quer nas atitudes, quer nos comportamentos dos estudantes de Medicina, e acredita que individualizem e medicalizem a deficiência, enfocando principalmente as dificuldades, sem expor as estratégias de enfrentamento ou habilidades desenvolvidas pelas pessoas com deficiência em seu cotidiano. Sublinha que esses exercícios não simulariam uma deficiência, mas apenas alguns efeitos de apresentar, temporariamente, uma deficiência, pois a experiência da deficiência permeia toda a vida da pessoa. Essas vivências não dariam conta dos aspectos sociais e psicológicos.

As pessoas com deficiência, seus familiares ou cuidadores vêm participando de forma passiva ou ativa, isto é, apenas enquanto pacientes observados pelos estudantes nas consultas em centros de reabilitação ou outros cenários de atenção à saúde; PCDs e seus familiares ou cuidadores falando sobre como é viver com deficiência aos estudantes em sala de aula; como pacientes padronizados; como pacientes padronizados educadores, palestrantes e tutores de oficinas ${ }^{30,25,24}$. Tanto na Universidade de Bristol como na Tufts, na Universidade de Londres e na de Buffalo, também participaram do planejamento das atividades, da elaboração de instrumento de avaliação do desempenho dos estudantes de Medicina, das habilidades, conhecimentos e atitudes que os futuros médicos devem possuir; e da implementação do currículo.

Mesmo que inicialmente tenham surgido como proposta de um ou outro professor, a partir de 2000 começam a surgir relatos de atividades resultantes de projetos institucionais, como na Universidade Tulane, em Nova Orleans ${ }^{18}$, na Universidade de Bristol ${ }^{36}$ e na Universidade Tufts ${ }^{25}$. Antes contemplando apenas um tipo de deficiência - como, por exemplo, anamnese de pessoas com deficiência intelectual nas aulas de Psiquiatria -, passam a ser construídas atividades que consideram vários tipos de deficiência ${ }^{18}$. No lugar de atividades isoladas e de curta duração, a partir de 2009 passam a ser distribuídas ao longo do curso médico ${ }^{19,37,38,39}$. 


\section{DISCUSSÃO}

Todos os exemplos foram encontrados fora do Brasil, devendo-se contextualizar as experiências, seja no aspecto cultural, seja na estrutura dos currículos, ou na duração do curso - quatro anos nas escolas americanas. A inclusão se deu ora ao longo do curso, nas disciplinas já existentes e em caráter obrigatório, ora como atividades pontuais, com participação voluntária, das quais participou um número reduzido de estudantes.

Atitudes, conhecimentos e habilidades são igualmente importantes, embora as escolas médicas tendam a dar mais valor à aquisição de conhecimentos. Não resta dúvida de que o contato direto dos estudantes de Medicina com pessoas com deficiência faz toda a diferença na forma como os estudantes as veem. Entretanto, para que isso ocorra, estas devem representar imagens não estereotipadas e mostrar sua expertise, inclusive participando como professoras.

A verdadeira inclusão das pessoas com deficiência no ensino médico deve se dar nas várias disciplinas ao longo do curso, incluindo as da área clínica e cirúrgica. Discutir temas ligados à deficiência em uma disciplina optativa ou em único momento no curso pode ser um primeiro passo, mas não deve ser o único. $\mathrm{O}$ cuidado no pré-natal e parto de gestantes com deficiência, por exemplo, deve ser ensinado na disciplina de Obstetrícia; nas aulas de Semiologia, os alunos devem aprender como se comunicar com pacientes surdos ou com dificuldades na fala, ou como reconhecer uma doença com base em sinais e sintomas que se apresentam de forma distinta em pessoas com lesão medular. Mais ainda, é importante que a inclusão da atenção à saúde das pessoas com deficiência seja planejada atendendo a uma lógica e que os professores/médicos tenham sua prática pautada pelo modelo biopsicossocial de perceber a deficiência. Por último, é indispensável o contato direto com pessoas com deficiência no papel de educadores, participando em conversas, como palestrantes ou pacientes padronizados.

\section{CONCLUSÃO}

Esperamos que esta pesquisa sirva de apoio a esforços para preparar melhor futuros médicos para cuidar de pacientes com deficiência. Entretanto, é preciso sensibilizar e comprometer professores e gestores das escolas médicas, assim como os ministérios da Educação e da Saúde para que a inclusão do tema deficiência seja uma realidade também em nosso país, contribuindo para a qualidade do cuidado prestado às pessoas com deficiência.

Essa qualidade não pode ser garantida apenas com profissionais sensibilizados, hábeis e com conhecimento sobre as necessidades das pessoas com deficiência; é preciso também que os equipamentos e os serviços de saúde sejam desenhados partindo do conceito de desenho universal. Se não temos como atuar em todas as frentes, sempre podemos dar um primeiro passo.

\section{REFERÊNCIAS}

1. World Health Organization. World Report on Disability. 2011 [capturado em: 13 ago 2012]. Disponível em: http:// www.who.int.

2. Iezzoni LI, O'day BL, Killeen M, Harker H. Communicating about health care: observations from persons who are deaf or hard of hearing. Annals of Internal Medicine 2004;140(5):356-362.

3. Brasil. Ministério da Saúde. Portaria MS n¹.060 de 5 de junho de 2002. Aprova, na forma de anexo desta Portaria, a Política Nacional de Saúde da Pessoa Portadora de Deficiência. Diário Oficial da República Federativa do Brasil. Brasília, jun. 2002; Seção 1, p.21.

4. Brasil. Ministério da Educação. Resolução CNE/CES nº 4, de 7 de novembro de 2001. Institui Diretrizes Curriculares Nacionais do Curso de Graduação em Medicina. Diário Oficial da União, Brasília, 9 de novembro de 2001. Seção 1, p. 38.

5. Costa LSM, Silva NCZ. Developing medical students' attitudes, knowledge and skills in health care of deaf people. Interface Comunicação Saúde Educação 2012; 16(43):545555.

6. Costa LSM. Deficiência e Educação Médica. In: Anais do 43. Congresso Brasileiro de Educação Médica; 2005; Natal, Brasil. p.556.

7. Costa LSM. Educação em Saúde para a comunidade surda. In: Fórum INES; vol.19/20. Rio de Janeiro: INES; 2010. p.35-37.

8. Costa LSM, Almeida, RCN de, Mayworn MC, Alves PTF, Bulhões PAM de, Pinheiro VM. O atendimento em saúde através do olhar da pessoa surda: avaliação e propostas. Revista Brasileira de Clínica Médica 2009; 7(3):166-170.

9. Costa LSM, Botelho IV, Souza LS. Abordagem do tema deficiência na literatura médica. In: Anais do 46. Congresso Brasileiro de Educação Médica; 2008; Salvador, Brasil. Salvador: ABEM; 2008. p. 246.

10. Vieira VHR, Paixão DMRI, Silva ABRL, Abrahão SC, Fernandes BS, Almeida MJ. Atenção Integral à Saúde da Pessoa com Deficiência: a teoria na prática. In: Anais do 46. Congresso Brasileiro de Educação Médica; 2008; Salvador, Brasil. Salvador: ABEM; 2008. p.65.

11. Vieira VHR, Leal JM, Martinelli MB, Chelini PR, Nascimento RO, Oliveira RSFO, cardoni TS, Silva MR, Amin BO, Sou- 
za PMT. Abordagens de ensino e atitudes de estudantes de medicina em relação a pessoas com deficiência. In: Anais do 44. Congresso Brasileiro de Educação Médica; 2006; Gramado, Brasil. Rio Grande do Sul: ABEM; 2006. p.382.

12. Ferreira CL, Koifman L, Costa LSM. Relação médico-paciente e a perspectiva inclusiva da pessoa com deficiência. In: Anais do 43. Congresso Brasileiro de Educação Médica; 2005; Natal, Brasil. p. 570.

13. Galil A, Margolis CZ, Glick S. The disabled in the community. Academic Medicine 1995; 70(5): 437-438.

14. Conill A. Living with disability: a proposal for medical education. Journal of the American Medical Association 1998; 279(1):83.

15. Crotty M, Finucane P, Ahern M. Teaching medical students about disability and rehabilitation: methods and student feedback. Medical Education 2000; 34(8):659-664.

16. Sabharwal S, Sebastian JL, Lanouette M. An educational intervention to teach medical students about examining disabled patients. Journal of the American Medical Association 2000; 284(9):1080-1081.

17. Graham CL, Brown RS, Zhen H, McDermott S. Teaching medical students about disability in family medicine. Family Medicine 2009; 41(8):542-544.

18. Saketkoo 1, Anderson D, Rice J, Rogan A, Lazarus CJ. Effects of a disability awareness and skills training workshop on senior medical students as assessed with self ratings and performance on a standardized patient case. Teaching and Learning in Medicine 2004; 16(4):345-354.

19. Symons AB, McGuigan D, Akl EA. A curriculum to teach medical students to care for people with disabilities: development and initial implementation. BMC Medical Education 2009; 9(78) [capturado em: 15 mar. 2011]. Disponível em: http:/ / www.biomedcentral.com/1472-6920/9/78.

20. Thistlethwaite JE, Ewart BR. Valuing diversity: helping medical students explore their attitudes and beliefs. Medical Teacher 2003; 25(3):277-281.

21. Mitchell KR, Hayes M, Gordon J, Wallis B. An investigation of the attitudes of medical students to physically disabled people. Medical Education 1984; 18(1):21-23.

22. Jones $\mathrm{P}$, Donald M. Teaching medical students about children with disabilities in a rural setting in a school. BMC Medical Education 2007; 7(12) [capturado em: 15 mar. 2011]. Disponível em: http://www.biomedcentral. com/1472-6920/7/12

23. Martin HL, Rowell MM, Reid SM, Marks MK, Reddihough DS. Cerebral palsy: what do medical students know and believe. Journal of Paediatrics and Child Health 2005; 41(12):43-47.
24. Hall I, Hollins S. Changing medical students' attitudes to learning disability. Psychiatric Bulletin 1996; 20: 429 - 430.

25. Minihan PM, Bradshaw YS, Long LM, Altman W, Perduta-Fulginiti S, Ector J, Foran KL, Johnson L, Khan P, Sneirson R. Teaching about disability: involving patients with disabilities as medical educators. Disability Studies Quarterly Fall 2004;24(4).

26. Henley LD. A home visit programme to teach medical students about children with special needs. Medical Education 1999; 33(10):749 - 752.

27. Wells TPE, Byron MA, McMullen SHP, Birchall MA. Disability teaching for medical students: disabled people contribute to curriculum development. Medical Education 2002;36(8):788-792.

28. Sabharwal S, Bronwell AM. Objective assessment and structured teaching of disability etiquette. Academic Medicine 2001; 76(5):509.

29. Richard I, Compain V, Mouillie JM, Adès F, Garnier F, Dubas, F.; Saint-andré, J.P. Évaluation de l'attitudes vis-à-vis des personnes handicapées des étudiants en médecine de 3e et $4 \mathrm{e}$ année par le questionnaires. Annales de réadaptation et de medicine physique 2005 ; 48:662-667.

30. Minihan PM, Robey KL, Long-Bellil LM, Graham CL, Hahn JE, Woodard L, Eddey GE. Desired education outcomes of disability-relates training for the generalist physician: knowledge, attitudes, and skills. Academic Medicine 2011; 86(9):1171-1178.

31. Dugan A, Bradshaw YS. How do I ask about your disability? An examination of interpersonal communication processes between medical students and patients with disabilities. Journal of Health Communication 2010; 15(3):334-350.

32. Khan P. Teaching Tomorrow's docs. New Mobility 2003; 9:45-48.

33. Eddey GE, Robey KL. Considering the Culture of Disability in Cultural Competence Education. Academic. Medicine 2005; 80(7):706-712.

34. Amosun SL, Volmink L, Rosin R. Perceived images of disability: the reflections of two undergraduate medical students in a university in South Africa on life in a wheelchair. Disability and Rehabilitation 2005; 27(16):961-966.

35. French S. Simulation exercises in disability awareness training: a critique. Disability, Handicap \& Society 1992; 7(3):257-266.

36. Byron M; Dieppe P. Educating health professionals abut disability: 'attitudes, attitudes, attitudes'. J. R. Soc. /med., 2000; 93 (8): 397-8.

37. Thacker A, Perez W; Crabble N; McCluskey C; Hollins S; Raji O. The Contribution of Actors with Intellectual Di- 
sabilities to the Training of Medical Students. Disponível em: <hrrp:www.intellectualdisability.info/how-to../the-contribution-ofactors-with-intellectual-disabilities- to-the-training-of-medicalstudents $>$. Acessado em março de 2012.

38. Piachaud, J. Teaching learning disability to undergraduate medical students. Advances in Psychiatric Treatment, 2002; 8 (5): 334-341.

39. Tracy J, Iacono T. People with developmental disabilities teaching medical students - does it make a difference? Journal of Intellectual \& Developmental Disability 2008; 33(4):345-348.

\section{CONTRIBUIÇÃO DOS AUTORES}

Ambas as autoras participaram da coleta de dados, análise e redação do artigo.

\section{CONFLITO DE INTERESSES}

Não há conflito de interesses de qualquer das autoras.

\section{ENDEREÇO PARA CORRESPONDÊNCIA}

Luiza Santos Moreira da Costa

Rua Marquês do Paraná, 303. Prédio Anexo, 3ํandar.

Centro - Niterói

CEP 24030-210 RJ

E-mail: luiza.costa.psi@gmail.com 